\title{
Evidence of coupling between the thermal and nonthermal emission in the gamma-ray binary LS I +61 303
}

\author{
X. Paredes-Fortuny ${ }^{1}$, M. Ribó ${ }^{1, \star \star}$, V. Bosch-Ramon ${ }^{1}$, J. Casares $^{2,3}$, O. Fors ${ }^{4,1}$, and J. Núñez ${ }^{1,5}$ \\ 1 Departament d'Astronomia i Meteorologia, Institut de Ciènces del Cosmos, Universitat de Barcelona, IEEC-UB, \\ Martí i Franquès 1, 08028 Barcelona, Spain \\ e-mail: xparedes@am.ub.es \\ 2 Instituto de Astrofísica de Canarias, 38205 La Laguna, Santa Cruz de Tenerife, Spain \\ 3 Departamento de Astrofísica, Universidad de La Laguna, 38206 La Laguna, Santa Cruz de Tenerife, Spain \\ ${ }^{4}$ Department of Physics and Astronomy, University of North Carolina at Chapel Hill, Chapel Hill, NC 27599-3255, USA \\ 5 Observatori Fabra, Reial Acadèmia de Ciències i Arts de Barcelona, Rambla dels Estudis, 115, 08002 Barcelona, Spain \\ Received 18 November 2014 / Accepted 19 December 2014
}

\section{ABSTRACT}

\begin{abstract}
The gamma-ray binary LS I +61 303 is composed of a Be star and a compact companion orbiting in an eccentric orbit. Variable flux modulated with the orbital period of $\sim 26.5 \mathrm{~d}$ has been detected from radio to very high-energy gamma rays. In addition, the system presents a superorbital variability of the phase and amplitude of the radio outbursts with a period of $\sim 4.6 \mathrm{yr}$. We present optical photometric observations of LS I +61 303 spanning $\sim 1.5 \mathrm{yr}$ and contemporaneous $\mathrm{H} \alpha$ equivalent width $\left(E W_{\mathrm{H} \alpha}\right)$ data. The optical photometry shows, for the first time, that the known orbital modulation suffers a positive orbital phase shift and an increase in flux for data obtained 1-yr apart. This behavior is similar to that already known at radio wavelengths, indicating that the optical flux follows the superorbital variability as well. The orbital modulation of the $E W_{\mathrm{H} \alpha}$ presents the already known superorbital flux variability but shows, also for the first time, a positive orbital phase shift. In addition, the optical photometry exhibits a lag of $\sim 0.1-0.2$ in orbital phase with respect to the $E W_{\mathrm{H} \alpha}$ measurements at similar superorbital phases, and presents a lag of $\sim 0.1$ and $\sim 0.3$ orbital phases with respect noncontemperaneous radio and X-ray outbursts, respectively. The phase shifts detected in the orbital modulation of thermal indicators, such as the optical flux and the $E W_{\mathrm{H} \alpha}$, are in line with the observed behavior for nonthermal indicators, such as X-ray or radio emission. This shows that there is a strong coupling between the thermal and nonthermal emission processes in the gamma-ray binary LS I +61303 . The orbital phase lag between the optical flux and the $E W_{\mathrm{H} \alpha}$ is naturally explained considering different emitting regions in the circumstellar disk, whereas the secular evolution might be caused by the presence of a moving one-armed spiral density wave in the disk.
\end{abstract}

Key words. X-rays: binaries - X-rays: individuals: LS I +61 303 - binaries: close - stars: emission-line, Be - gamma rays: stars

\section{Introduction}

LS I +61 303 is one of the five gamma-ray binaries currently known (e.g., Dubus 2013; Paredes et al. 2013). It is composed of an optical star $(V \sim 10.7)$ with spectral type B0 Ve, therefore presenting a circumstellar disk, and a compact companion that is probably a pulsar orbiting in an eccentric orbit with $e$ in the range 0.54-72 (see Hutchings \& Crampton 1981; Paredes \& Figueras 1986; Casares et al. 2005; Dhawan et al. 2006; Aragona et al. 2009). The distance to the source is estimated to be $\sim 2 \mathrm{kpc}$ based on H I measurements (Frail \& Hjellming 1991). The binary system has an orbital period of $26.4960 \pm 0.0028 \mathrm{~d}$ (Gregory 2002), and variable flux modulated with the orbital period has been detected from radio to very high-energy gamma rays (e.g., Taylor \& Gregory 1982; Mendelson \& Mazeh 1989; Paredes et al. 1994, 1997; Abdo et al. 2009; Albert et al. 2009). In addition to the orbital modulation, LS I +61 303 also exhibits a superorbital modulation of radio outbursts in $\sim 4.6 \mathrm{yr}$ (first detected by Paredes 1987 and Gregory et al. 1989). Gregory (2002) found a superorbital period of $1667 \pm 8 \mathrm{~d}$ in the amplitude and in the orbital phase of the radio outbursts, leading to a drift of

\footnotetext{
* Tables 1 and 2 are only available at the CDS via anonymous ftp to cdsarc.u-strasbg.fr (130.79.128.5) or via

http://cdsarc.u-strasbg.fr/viz-bin/qcat?J/A+A/575/L6

$\star \star$ Serra Húnter Fellow.
}

the outburst maxima from orbital phase $\sim 0.4$ to $\sim 0.9$. For reference, the periastron passage takes place at orbital phase 0.23 0.28 (Casares et al. 2005; Aragona et al. 2009). The zero orbital and superorbital phases are set at JD 2443366.775 (Gregory 2002).

Zamanov et al. (2013) have recently detected the $\sim 4.6 \mathrm{yr}$ superorbital period in the equivalent width of the $\mathrm{H} \alpha$ emission line, confirming the earlier evidences reported in Zamanov et al. (1999). This suggests that the superorbital variability is related to periodic changes in the mass-loss rate of the Be star and/or variations in the circumstellar disk (see Massi \& Torricelli-Ciamponi 2014 and references therein for alternative interpretations). $\mathrm{Li}$ et al. (2012) discovered the superorbital modulation of LS I +61 303 in X-rays (3-30 keV), with a period compatible with that found by Gregory (2002) from radio measurements. Chernyakova et al. (2012) also reported the superorbital modulation of the X-ray emission, and using contemporaneous X-ray and radio observations showed that radio outbursts lag the X-ray outbursts by $\sim 0.2$ in orbital phase along the entire superorbital cycle. Ackermann et al. (2013) found a sinusoidal variability in the $>100 \mathrm{MeV}$ gamma-ray flux consistent with the radio superorbital period. Finally, Zaitseva \& Borisov (2003) found long-term variability in the mean $V$-band magnitude of $0.07 \mathrm{mag}$, but no search for superorbital variability was conducted. 
Table 3. Fitted sinusoidal parameters to the orbital variability of the optical photometry and the $E W_{\mathrm{H} \alpha}$ of LS I +61303 for the total data set (Fig. 1) and for the two different observational seasons (Fig. 2).

\begin{tabular}{lcccccccc}
\hline \hline Data set & MJD $^{\text {optical }}$ & $A^{\text {optical }}(\mathrm{mmag})$ & $\phi_{0}^{\text {optical }}$ & $C^{\text {optical }}(\mathrm{mag})$ & $\mathrm{MJD}^{E W_{\mathrm{H} \alpha}}$ & $A^{E W_{\mathrm{H} \alpha}(\AA)}$ & $\phi_{0}^{E W_{\mathrm{H} \alpha}}$ & $C^{E W_{\mathrm{H} \alpha}}(\AA)$ \\
\hline Total & $56139-56723$ & $28 \pm 3$ & $0.73 \pm 0.02$ & $10.701 \pm 0.002$ & $56110-56672$ & $1.2 \pm 0.2$ & $0.56 \pm 0.03$ & $11.5 \pm 0.2$ \\
Season 1 & $56139-56335$ & $29 \pm 3$ & $0.68 \pm 0.02$ & $10.704 \pm 0.003$ & $56110-56298$ & $1.4 \pm 0.3$ & $0.54 \pm 0.03$ & $11.2 \pm 0.2$ \\
Season 2 & $56594-56723$ & $24 \pm 4$ & $0.85 \pm 0.02$ & $10.689 \pm 0.003$ & $56603-56672$ & $1.3 \pm 0.3$ & $0.76 \pm 0.05$ & $13.0 \pm 0.3$ \\
\hline
\end{tabular}

Notes. The parameters are those of the functions: optical $m=-A \cos \left(2 \pi\left[\phi-\phi_{0}\right]\right)+C$ and $E W_{\mathrm{H} \alpha}=A \cos \left(2 \pi\left[\phi-\phi_{0}\right]\right)+C$. Phases were computed using an orbital period of $26.4960 \mathrm{~d}$ and phase zero at JD 2443366.775.

In this work, we present optical photometric observations of LS I +61 303 spanning 1.5 yr, and contemporaneous H $\alpha$ equivalent width $\left(E W_{\mathrm{H} \alpha}\right)$ observations. We report the discovery of an orbital phase shift and a variation in the orbitally modulated flux, very similar to the well-known superorbital behavior of the orbital modulation in radio. In addition, we also detect a phase shift in the orbital modulation of the $E W_{\mathrm{H} \alpha}$, to our knowledge reported here for the first time. We discuss the obtained results in the context of the radio and X-ray superorbital variability, and conclude that there is a strong coupling between the thermal and nonthermal emission in LS I +61 303.

\section{Observations and data reduction}

We performed optical photometric observations of LS I +61 303 with the robotic Telescope Fabra-ROA Montsec (TFRM; see Fors et al. 2013). The TFRM is installed at the Observatori Astronòmic del Montsec (Lleida, Spain). The main specifications are: corrector plate of $0.5 \mathrm{~m}$ aperture and $0.78 \mathrm{~m}$ primary mirror, refurbished Baker-Nunn Camera for routine CCD robotic observations, focal ratio $f / 0.96,4.4^{\circ} \times 4.4^{\circ}$ field of view with a pixel scale of 3.9"/pixel, passband filter SCHOTT GG475 $(\lambda>$ $475 \mathrm{~nm}$ ), and custom CCD based on FLI ProLine 16803 with quantum efficiency of $60 \%$ at $550 \mathrm{~nm}$ (Fors et al. 2013).

The observations span from 2012 July 31 to 2014 March 7 (2 seasons) with 71 nights of good data (preliminary results on season 1 data were presented in Paredes-Fortuny et al. 2014). We observed the target around 20 times per night with exposures of 5-10 s, as a compromise between good signal to noise ratio and avoiding the nonlinear CCD regime.

We conducted the data reduction and analysis using a pipeline developed in Python following these steps: standard calibration of the images using IRAF $^{1}$ (implemented through PyRAF $^{2}$ ), aperture photometry using the PHOT package from IRAF (PyRAF) with an aperture radius of 7.5 pixels, and correction of the lightcurves using a weighted average differential magnitude correction method based on Broeg et al. (2005). The lightcurve of LS I +61 303 has been corrected using 145 weighted reference stars up to 4 mag fainter than the target and as close as possible to it $\left(<0.25^{\circ}\right)$. Finally, we averaged the corrected magnitudes of the target on a nightly basis, and we used an artificial offset to a mean magnitude of 10.7 mag. The nightly photometric uncertainties are estimated as the standard deviation of the magnitudes of the target obtained from the individual images for each night. The obtained values are typically in the 5-10 mmag range.

We also considered contemporaneous $E W_{\mathrm{H} \alpha}$ data of LS I +61 303 obtained using FRODOspec on the robotic $2.0 \mathrm{~m}$

\footnotetext{
1 IRAF is distributed by NOAO, which is operated by AURA, under cooperative agreement with NSF.

2 PyRAF is a product of the Space Telescope Science Institute, which is operated by AURA for NASA.
}

Liverpool telescope at the Observatorio del Roque de Los Muchachos (La Palma, Spain). The data span from 2012 July 2 to 2014 January 15 with 104 measurements, with one $600 \mathrm{~s}$ exposure per night. The data have been obtained with the same setup, and reduced in the same way, as in Casares et al. (2012). We assumed that the uncertainties of the $E W_{\mathrm{H} \alpha}$ are at the $10 \%$ level. The adopted convention is positive $E W$ for emission. The data (Tables 1 and 2) are available at the CDS.

\section{Results}

The optical photometric lightcurve and $E W_{\mathrm{H} \alpha}$ of LS I +61 303 as a function of MJD and folded with the orbital phase are shown in Fig. 1. As can be seen, the data cover two different seasons (centered in autumn) and show the already known orbital variability (see, e.g., Zamanov et al. 2014). The folded lightcurves for the first and second season and their sinusoidal fits are shown in Fig. 2. The fitted sinusoidal parameters (amplitude, zero phase, and mean value) for the different data sets, considering the data uncertainties, are quoted in Table 3. The $1 \sigma$ uncertanties in the fitted parameters quoted in Table 3 are estimated from the covariance matrix.

The fits to the orbital variability of the optical photometry reveal, for the first time, a positive orbital phase shift of $0.16 \pm 0.03$ at $5.2 \sigma$ confidence level (c.l.) and an increase in the average optical flux of $15 \pm 4$ mmag at $4.2 \sigma$ c.l. between the two observational seasons 1-yr apart. These trends are reminiscent of the superorbital trends found at radio and X-ray wavelengths, and indicate that the optical flux follows the superorbital variability as well. We note that the folded lightcurves of the two individual seasons (see Fig. 2-left) show less scatter than the folded lightcurve including all the data (see Fig. 1), which span $\sim 0.35$ superorbital phases.

Similarly, the fits to the orbital variability of $E W_{\mathrm{H} \alpha}$ present for the first time a positive orbital phase shift of $0.22 \pm 0.05$ at $4.2 \sigma$ c.l. Further, we confirm the previously claimed superorbital modulation of the mean $E W_{\mathrm{H} \alpha}$, with a variation of $1.8 \pm 0.3 \AA$ at $5.7 \sigma$ c.l., between our two observational seasons ${ }^{3}$. In addition, the orbital modulation of the optical photometry exhibits a lag of $\sim 0.1-0.2$ in orbital phase with respect to the orbital modulation of $E W_{\mathrm{H} \alpha}$ at similar superorbital phases (see Fig. 2 and Table 3).

To better display the superorbital variability, color maps of the optical photometry and $E W_{\mathrm{H} \alpha}$ as a function of the orbital phase and superorbital cycle are shown in Fig. 3. The dotted red and blue lines represent the orbital phase drifts of the corresponding maxima along the superorbital cycle for the contemporaneous optical photometry and $E W_{\mathrm{H} \alpha}$ presented here (superorbital cycle 7). The contemporaneous radio and X-ray fluxes of the previous superorbital cycle (6) from Chernyakova et al. (2012) are shown as black and green dotted lines. The

\footnotetext{
3 Despite the relatively poor sampling at equivalent superorbital phases, the Zamanov et al. (2013) data reveal a compatible behavior.
} 

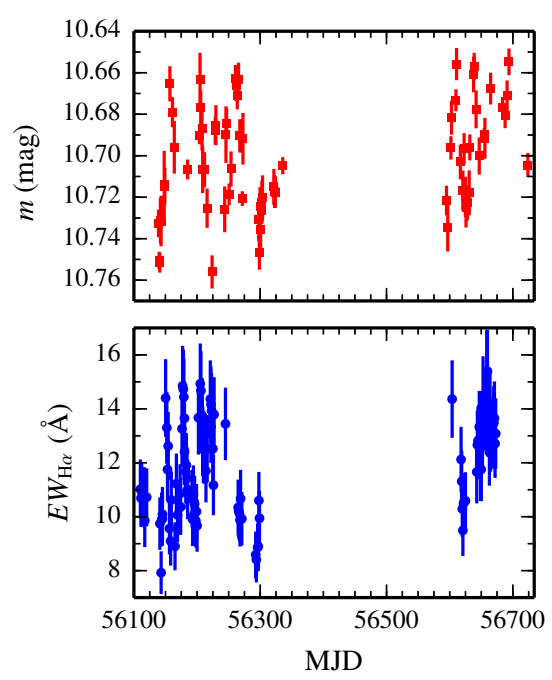
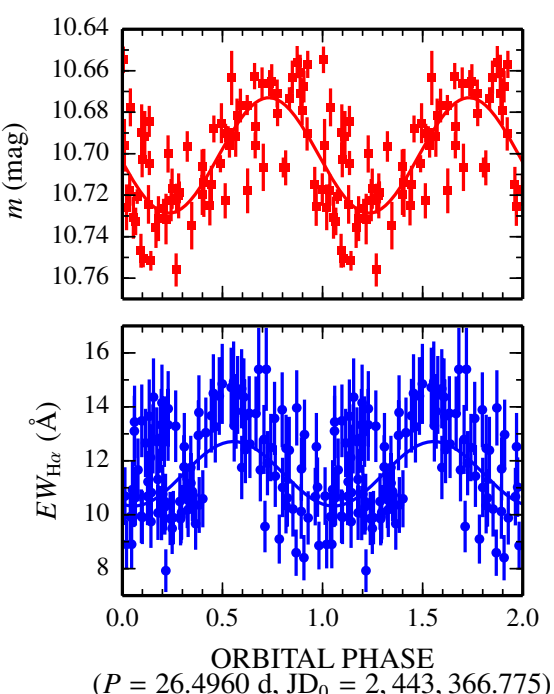

Fig. 1. Optical photometric lightcurve (top, red) and $E W_{\mathrm{H} \alpha}$ (bottom, blue) of LS I +61 303 plotted as a function of MJD (left) and folded with the orbital phase (right). Error bars represent $1 \sigma$ uncertainties. The solid curves in the right-hand panels represent sinusoidal fits to the orbital variability, whose parameters can be found in Table 3. Two cycles are displayed for clarity.
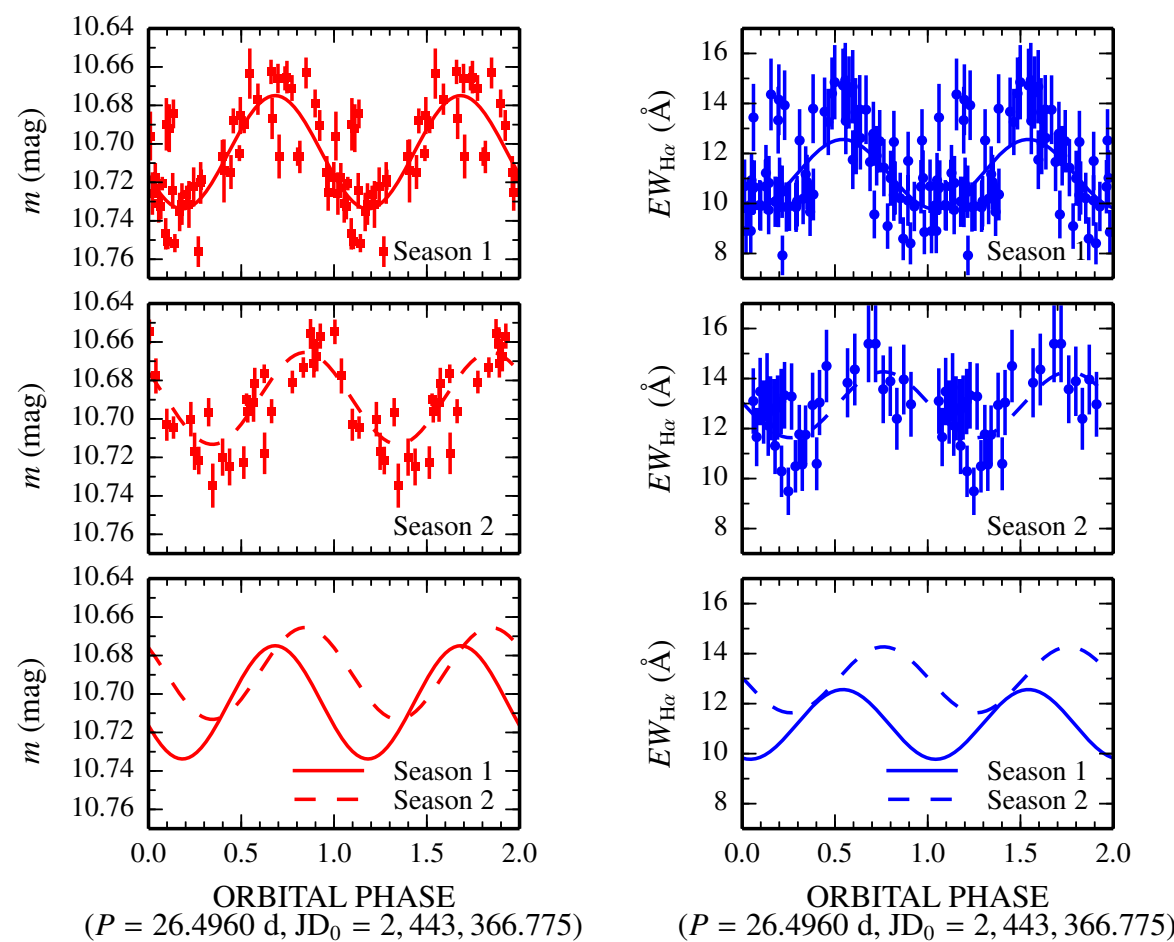

Fig. 2. Optical photometric lightcurve (left, red) and $E W_{\mathrm{H} \alpha}$ (right, blue) of LS I +61303 folded with the orbital phase for the first (top) and second (center) seasons, and their sinusoidal fits to the orbital variability (bottom; see Table 3). Error bars represent $1 \sigma$ uncertainties. Two cycles are displayed for clarity. The shifts in the orbital modulations between both seasons are clear.

optical photometric observations show a lag of $\sim 0.1$ in orbital phase with respect to the radio outburst, and $\sim 0.3$ with respect to the X-ray outbursts for equivalent superorbital phases one cycle apart. The $E W_{\mathrm{H} \alpha}$ maxima occur at orbital phases similar to the radio outbursts for similar superorbital phases one cycle apart, while in the averaged data of Zamanov et al. (2013, 2014), the maxima occur during the rising of the radio flux density. The different slopes in Fig. 3 might suggest a change in the behavior of the superorbital modulation between different superorbital cycles, although we caution that the results are still compatible at $2 \sigma$ c.l.

\section{Discussion}

The observations of LS I +61 303 reported here show, for the first time, orbital phase shifts in the maxima of the orbitally modulated optical flux and $E W_{\mathrm{H} \alpha}$ for different phases of the superorbital cycle (see Figs. 2 and 3 and Table 3 ). These results extend previously reported links between the $\mathrm{H} \alpha$ emission and multiwavelength properties but obtained from the average orbital modulation, i.e., without considering the superorbital variability (see, e.g., Zamanov et al. 2014). The results reported here are 

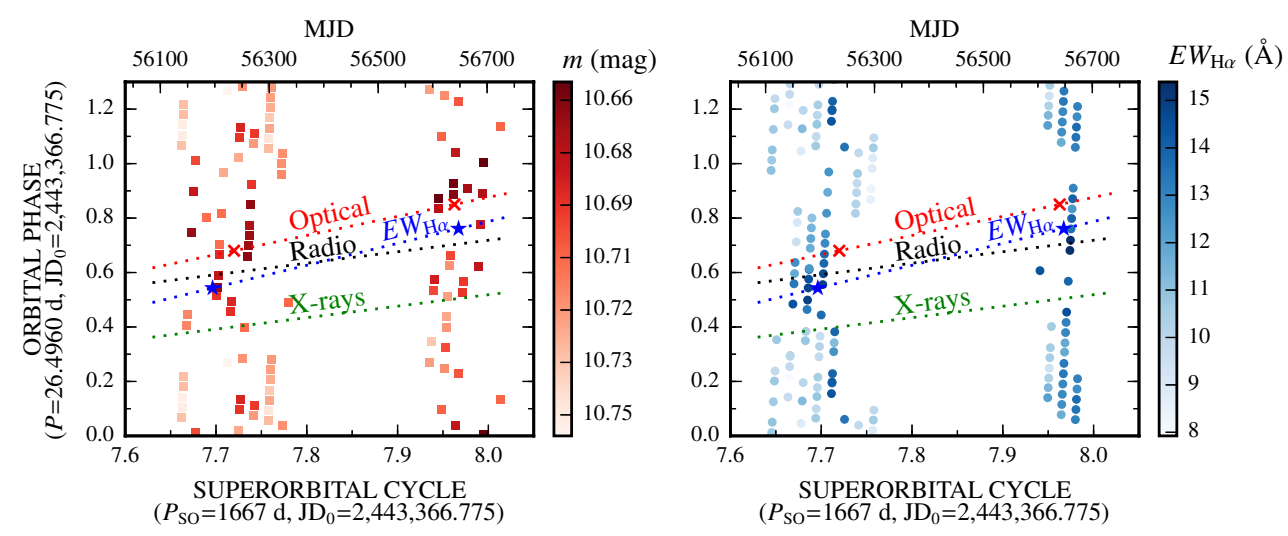

Fig. 3. Color maps of the optical photometry (left) and $E W_{\mathrm{H} \alpha}($ right) from LS I +61303 as a function of the orbital phase and superorbital cycle. The red crosses and blue stars correspond to the phases of the maxima of the sinusoidal fits to the orbital variability of the optical photometry and $E W_{\mathrm{H} \alpha}$, respectively, for the first and second seasons (see Table 3). The dotted color lines represent the orbital phase drift of the emission peaks caused by the superorbital variability for the optical photometry (red), $E W_{\mathrm{H} \alpha}$ (blue), radio (black), and X-rays (green). The radio and X-ray drifts are taken from Fig. 3 of Chernyakova et al. (2012) using data of the previous superorbital cycle.

now discussed in the context of the known multiwavelength behavior of the orbital and superorbital variability of the source.

The orbital variability of the gamma-ray binary LS I +61 303 is wavelength dependent. After periastron at phase $0.23-0.28$, there is first a maximum in X-rays (good tracer of the nonthermal emission), followed by a maximum in $E W_{\mathrm{H} \alpha}$ (tracer of the outer disk conditions) and in radio emission (produced mainly outside the binary system). Finally, a maximum in optical flux is observed, with a $65 \%$ contribution from the Be star plus $35 \%$ from the inner circumstellar disk (Casares et al. 2005). The circumstellar disk is likely to be perturbed and/or partially disrupted by tidal forces and the putative pulsar wind ram pressure. These would trigger significant changes in the structure of the Be disk, especially around periastron passage, reducing its emitting area and total optical emission. This is approximately what is observed in the data from season 1, but the orbital phase shift in season 2 clearly shows that there is more than purely orbit-induced variability. In fact, the superorbital variability observed in $E W_{\mathrm{H} \alpha}$ has been associated with periodic changes on the Be star envelope and its circumstellar disk (e.g., Zamanov et al. 1999), which would trigger the superorbital variability observed at other wavelenghts. If this is the case, since the orbital modulation seen in X-rays or radio suffers a phase drift along the superorbital cycle, we should detect the same effect in optical flux and $E W_{\mathrm{H} \alpha}$, as reported here for the first time. Thus, it is clear that there is a strong empirical coupling between the thermal (optical) and the nonthermal (X-ray and radio) emission in the gamma-ray binary LS I +61 303 at both orbital and superorbital scales.

The orbitally modulated optical flux and $E W_{\mathrm{H} \alpha}$ present different behavior between them. The optical flux shows $\sim 0.06 \mathrm{mag}$ modulation, representing $\sim 6 \%$ in total flux or $\sim 16 \%$ in disk flux (and probably projected area), while $E W_{\mathrm{H} \alpha}$ shows $\sim 30 \%$ variability. This implies that external parts of the disk are more perturbed or disrupted than the inner parts of the disk, as one would expect if these perturbations (or disruptions) are due to the influence of the compact object as it approaches periastron (where the perturbations are caused by tidal forces and/or ram pressure). In addition, the $\sim 0.1-0.2$ phase lag implies that the external parts are perturbed or disrupted before the internal parts, but they recover earlier as well, probably building up from material shocked before periastron passage. The inner disk would only recover close to, or even after, apastron. The secular evolution of this behavior along the superorbital cycle could be due to the presence of a moving one-armed spiral density wave in the disk, as suggested by Negueruela et al. (1998) for traditional Be/X-ray binaries.

Acknowledgements. The authors acknowledge support of the TFRM team for preparing and carrying out the optical photometric observations. We thank useful discussions with Josep M. Paredes and Ignacio Negueruela. We acknowledge support by the Spanish Ministerio de Economía y Competitividad (MINECO) under grants AYA2013-47447-C3-1-P, AYA2010-18080, and FPA2013-48381C6-6-P. This research has been supported by the Marie Curie Career Integration Grant 321520. X.P.-F. acknowledges financial support from Universitat de Barcelona and Generalitat de Catalunya under grants APIF and FI (2014FI_B 01017), respectively. V.B.-R. acknowledges financial support from MINECO and European Social Funds through a Ramón y Cajal fellowship.

\section{References}

Abdo, A. A., Ackermann, M., Ajello, M., et al. 2009, ApJ, 701, L123

Ackermann, M., Ajello, M., Ballet, J., et al. 2013, ApJ, 773, L35

Albert, J., Aliu, E., Anderhub, H., et al. 2009, ApJ, 693, 303

Aragona, C., McSwain, M. V., Grundstrom, E. D., et al. 2009, ApJ, 698, 514

Broeg, C., Fernández, M., \& Neuhäuser, R. 2005, Astron. Nachr., 326, 134

Casares, J., Ribas, I., Paredes, J. M., Martí, J., \& Allende Prieto, C. 2005, MNRAS, 360, 1105

Casares, J., Ribó, M., Ribas, I., et al. 2012, MNRAS, 421, 1103

Chernyakova, M., Neronov, A., Molkov, S., et al. 2012, ApJ, 747, L29

Dhawan, V., Mioduszewski, A., \& Rupen, M. 2006, in Proc. VI Microquasar Workshop: Microquasars and Beyond, PoS(MQW6)052

Dubus, G. 2013, A\&ARv, 21, 64

Fors, O., Núñez, J., Muiños, J. L., et al. 2013, PASP, 125, 522

Frail, D. A., \& Hjellming, R. M. 1991, AJ, 101, 2126

Gregory, P. C. 2002, ApJ, 575, 427

Gregory, P. C., Xu, H.-J., Backhouse, C. J., \& Reid, A. 1989, ApJ, 339, 1054

Hutchings, J. B., \& Crampton, D. 1981, PASP, 93, 486

Li, J., Torres, D. F., Zhang, S., et al. 2012, ApJ, 744, L13

Massi, M., \& Torricelli-Ciamponi, G. 2014, A\&A, 564, A23

Mendelson, H., \& Mazeh, T. 1989, MNRAS, 239, 733

Negueruela, I., Reig, P., Coe, M. J., \& Fabregat, J. 1998, A\&A, 336, 251

Paredes, J. M. 1987, Ph.D. Thesis, University of Barcelona

Paredes, J. M., \& Figueras, F. 1986, A\&A, 154, L30

Paredes, J. M., Marziani, P., Marti, J., et al. 1994, A\&A, 288, 519

Paredes, J. M., Marti, J., Peracaula, M., \& Ribó, M. 1997, A\&A, 320, L25

Paredes, J. M., Bednarek, W., Bordas, P., et al. 2013, Astropart. Phys., 43, 301

Paredes-Fortuny, X., Ribó, M., Fors, O., Núñez, J., \& Bosch-Ramon, V. 2014, Int. J. Mod. Phys. Conf. Ser., 28, 60197

Taylor, A. R., \& Gregory, P. C. 1982, ApJ, 255, 210

Zaitseva, G. V., \& Borisov, G. V. 2003, Astron. Lett., 29, 188

Zamanov, R. K., Martí, J., Paredes, J. M., et al. 1999, A\&A, 351, 543

Zamanov, R., Stoyanov, K., Martí, J., et al. 2013, A\&A, 559, A87

Zamanov, R., Martí, J., Stoyanov, K., Borissova, A., \& Tomov, N. A. 2014, A\&A, 561, L2 